\title{
Date of Additional Surgery for New Tumor Event: Loco-Regional
}

National Cancer Institute

\section{Source}

National Cancer Institute. Date of Additional Surgery for New Tumor Event: Loco-

Regional. NCl Thesaurus. Code C160439.

Date of additional surgery for the new loco-regional tumor event. 